\title{
Arbor
}

\section{La Flota, razón de ser de la Armada}

\author{
José Antonio Balbás Otal
}

Arbor CLXXIII, 682 (Octubre 2002), 281-299 pp.

El título de este artículo expresa con suficiente claridad el alcance de su contenido. La Flota es la expresión material del poder naval con que cuenta la Armada. Sus dotaciones, buques de superficie, submarinos, aeronaves, medios de guerra de minas, fuerzas de Infantería de Marina, centros de adiestramiento e instalaciones navales en tierra, constituyen el núcleo fundamental de la Fuerza Naval.

A lo largo de su exposición, el Almirante de la Flota nos describe detalladamente la organización, composición y cometidos de las diferentes unidades, agrupaciones y dependencias que están bajo sus órdenes, poniendo especial énfasis en sus capacidades, estructura operativa y participación en ejercicios $y$ operaciones de carácter nacional e internacional. Asimismo, hace referencia a la contribución a la OTAN y a otras fuerzas multinacionales, y a la participación más reciente de unidades de la Flota en operaciones reales.

El artículo es una excelente síntesis para quien desee conocer cómo es y qué hace la Flota, cuál es su futuro en cuanto a incorporación de nuevas unidades, y qué procesos de reestructuración se están llevando a cabo, incluída la reciente creación de un Cuartel General Marítimo de Alta Disponibilidad, dentro de la estructura de fuerzas de la OTAN.

\section{Introducción}

La Flota es el núcleo fundamental de la Fuerza de la Armada y está constituida por el conjunto de buques de superficie, submarinos, aeronaves, medios de guerra de minas, fuerzas de Infantería de Ma- 
rina, centros de adiestramiento e instalaciones navales en tierra, puestos bajo las órdenes de un Almirante.

Es por tanto, la Flota, el componente más relevante del poder naval con que cuenta la Armada para cumplir los siguientes objetivos marítimos de la Defensa Nacional:

- Proteger los intereses nacionales en el ámbito marítimo.

- Participar en fuerzas multinacionales, dentro de la seguridad compartida de España con sus socios y aliados.

- Ser un instrumento clave de la política exterior

- Proyectar el poder naval sobre tierra.

- Conseguir el control del mar en los espacios geoestratégicos de interés nacional.

- Apoyar las actividades gubernamentales

- Colaborar con otros organismos del Estado, y con las Fuerzas y Cuerpos de Seguridad en situaciones de catástrofe y salvamento marítimo.

- Apoyar las misiones humanitarias y de paz.

La Flota, al igual que el resto de los organismos implicados en la Defensa de España, no ha sido ajena a los numerosos e importantes cambios sociales, estratégicos, legislativos y tecnológicos que han afectado profundamente a las Fuerzas Armadas en los últimos tiempos. Unos cambios que han obligado a modificar o adaptar la organización, las estructuras, los procesos de trabajo e incluso las formas de dirigir y mandar. En el caso concreto de la Flota los cambios se han centrado teniendo en cuenta los siguientes aspectos:

- La variación de la situación estratégica mundial a partir de la desmembración de la URSS, y muy especialmente a raíz de los acontecimientos del 11S, que ponen de manifiesto la aparición de riesgos asimétricos, entre los que destaca con fuerza el terrorismo internacional.

- La plena integración de España en la OTAN, y como consecuencia de ella, el desarrollo de un Cuartel General Marítimo de Alta Disponibilidad HRF (M) SPHQ, basado en un estado mayor multinacional y un buque de mando, el Castilla, puesto a disposición de la Alianza.

- La adaptación al proceso de plena profesionalización de marineros y soldados, que ha hecho que sus unidades tengan dotaciones totalmente profesionales. 


\section{El Almirante de la Flota}

El mando de la Flota lo ejerce un Almirante del Cuerpo General de la Armada, en situación de servicio activo, con la denominación de Almirante de la Flota (ALFLOT), y su campo de actividad prioritario es el adiestramiento de las unidades.

En este ámbito, dos son las misiones del ALFLOT: una permanente, que se ocupa de la preparación de la Flota, la moral de sus dotaciones y el ejercicio de la autoridad disciplinaria que le corresponde; y otra eventual, de conducción de las operaciones, ya sean de carácter nacional o multinacional, específico o conjunto.

La misión de preparación de la Flota abarca el conjunto de todas las actividades dirigidas a adiestrar, evaluar y alistar todos los medios y unidades a sus órdenes para que estén en condiciones de alcanzar eficazmente sus objetivos, con especial atención a los despliegues en teatros alejados y de considerable duración. Para ello, el ALFLOT tiene asignados los siguientes cometidos:

- Obtener, en todo momento, la máxima eficacia de los medios puestos bajo su mando, velando por la moral y disciplina de su personal.

- Planificar, supervisar y evaluar el adiestramiento individual y colectivo, tanto básico como avanzado, de las unidades de la Flota, de sus mandos subordinados y órganos de apoyo.

- Determinar las necesidades, fijar las prioridades y controlar los recursos económicos, de personal y de material que tenga asignados.

- Efectuar el seguimiento de aquellos factores que afecten a la eficacia, al personal y al material de las unidades de la Flota, coordinando cuando sea necesario con otras autoridades de la Armada.

- Formular las propuestas de modificación y de modernización de las unidades de la Flota.

- Sancionar la doctrina táctica de la Armada y los procedimientos de empleo táctico de unidades, y controlar su cumplimiento.

En cuanto a la misión eventual como conductor de operaciones, sus cometidos son:

- Participar en el planeamiento operativo y desarrollar los planes operativos y de contingencia que le correspondan.

- Ejercer las responsabilidades que se le asignen en los planes operativos nacionales, incluyendo la vigilancia y la patrulla marítima. 
Asumir la conducción de las operaciones y ejercer el mando operativo de las fuerzas asignadas.

Por último, es importante resaltar que además de los cambios ya mencionados y que han tenido una influencia en la Flota en general, el Almirante de la Flota ha ido asumiendo nuevas responsabilidades a lo largo de los últimos años que han dado lugar a cometidos específicos. Entre esas responsabilidades cabe destacar las siguientes:

- Asumir, periódicamente, el mando de la Fuerza Marítima Europea EUROMARFOR, un mando que corresponde por turno entre los Almirantes de la Flota de Francia, Italia, Portugal y España.

- Aportar el componente nacional a la Fuerza Anfibia italo-española SIAF.

- Elaborar y experimentar la doctrina táctica de la Armada.

- El mando de la Flotilla de Medidas Contra Minas.

\section{Estructura orgánica}

La Flota tiene una estructura básica similar a la de la Armada, con sus tres componentes principales: el Cuartel General, la Fuerza y el Apoyo a la Fuerza.

\section{El Cuartel General}

Situado en la Base Naval de Rota, Cádiz, el Cuartel General de la Flota está formado por un conjunto de órganos de asistencia al ALFLOT, cuyas funciones principales son las de apoyo a la decisión, dirección, administración y control. En concreto, incluye una serie de organismos dependientes directamente del Almirante de la Flota, como son el Estado Mayor, la Asesoría Jurídica, la Intervención Delegada, Intendencia, Sanidad, Oficina de Relaciones Públicas, Asistencia Religiosa y Ayudantía Mayor.

Para auxiliar en sus cometidos al Almirante de la Flota, el Cuartel General cuenta además con las siguientes instalaciones:

- Un Centro de Conducción de Operaciones Marítimas COM, ubicado en el propio Cuartel General, y cuya función principal es la de facilitar el planeamiento y conducir las operaciones de las unidades de la Flota desde un centro en tierra. 
Para poder llevar a cabo esta función, el centro cuenta con avanzados sistemas de mando y control que facilitan la evaluación y la toma de decisiones mediante el enlace del Cuartel General con los buques en la mar, con otros centros de operaciones nacionales, ya sean navales, terrestres o aéreos, y con los cuarteles generales de la OTAN o de fuerzas multinacionales directa o indirectamente implicados en las operaciones.

- Una Agencia de Control Aéreo Marítimo MACA, reconocida por la OTAN, para operar tanto en el Atlántico como en el Mediterráneo.

- Un Centro de Información, Gestión y Administración de la Flota CIGAFLOT.

- Un Centro de Comunicaciones CECOM y un Centro de Inteligencia Operativa CIO.

\section{La Fuerza}

La razón de ser de la Armada es la existencia y eficacia de la Fuerza, cuya organización ha sufrido una reciente remodelación llevada a cabo en el segundo semestre del año 2001.

La Flota dispone en esencia de una estructura orgánica permanente, concebida básicamente para la preparación de la Fuerza Naval, y desde la que se puede generar eventualmente cualquier estructura operativa de carácter temporal para el empleo de la Fuerza y adecuada a la misión que se le asigne.

En la nueva estructura orgánica de la Fuerza se encuadran:

- El Cuartel General Marítimo Español de Alta Disponibilidad, que constituye una unidad orgánica más, mientras no se encuentre inmerso en operaciones o ejercicios.

- Agrupaciones orgánicas tipo Grupos, Flotillas y Escuadrillas.

- Unidades independientes tipo Buques Logísticos.

- La Brigada de Infantería de Marina.

\section{El Cuartel General Marítimo Español de Alta Disponibilidad HRF (M) SPHQ}

El Cuartel General Marítimo Español de Alta Disponibilidad es un estado mayor naval, multinacional, capaz de intervenir, a las órdenes de su Comandante COMARFOR -un Vicealmirante del Cuerpo General-, 
en operaciones conjuntas y combinadas, y que en la mar operará normalmente a bordo de su buque de mando, el LPD Castilla.

Este Cuartel General se sitúa en la categoría de las fuerzas denominadas NATO Assigned, es decir, un medio nacional existente, que se asignará cuando se requiera, a un mando OTAN, y del modo que se determine en un acuerdo técnico que se encuentra en proceso de desarrollo. Por tanto, es un medio nacional ofrecido a la Alianza y no de la Alianza. Está también a disposición de la Unión Europea y se podrá poner a disposición de aquellas organizaciones internacionales que se determinen.

Como unidad incluida en la estructura orgánica de la Fuerza, el cometido del COMARFOR será el alistamiento y preparación de su Cuartel General de forma que pueda asumir el mando de una fuerza naval asignada, tanto nacional como aliada.

Además de mandar el Cuartel General Marítimo, el COMARFOR es la segunda autoridad de la Flota y por tanto el relevo natural del ALFLOT.

\section{Agrupaciones Orgánicas}

En ellas se busca la preparación óptima de las unidades de la Flota, con el fin último del alistamiento de sus unidades para la integración en las organizaciones operativas. En las agrupaciones orgánicas se realiza la preparación, el adiestramiento y la determinación de necesidades, tanto en puerto como en la mar, en maniobras o en ejercicios. Se clasifican en Grupos, Flotillas y Escuadrillas.

- Desde la última reorganización, en la Flota sólo existe un Grupo, denominado Grupo de Unidades de Proyección de la Flota GRUFLOT, a cuyo mando se encuentra un contralmirante denominado COMGRUFLOT. El grupo lo integran las unidades que materializan las capacidades aeronaval y anfibia de la Armada, es decir, el portaaviones Príncipe de Asturias, los buques anfibios tipo LPD Galicia y Castilla, este último especialmente preparado como buque de mando, y dos buques tipo LST Hernán Cortés y Pizarro, así como aquellos escoltas y buques logísticos que se determinen de acuerdo con la misión.

Los dos LST, aunque ya están en su último tercio de vida, cuentan todavía con una significativa capacidad operativa. Además de estas unidades, el GRUFLOT se completa con el Grupo 
Naval de Playa, que comprende todas las embarcaciones y medios auxiliares de desembarco con los que cuenta la Armada.

- Las Flotillas agrupan unidades de similares características y cometidos, para facilitar su adiestramiento y mantenimiento. En la actualidad existen tres: La Flotilla de Submarinos, la Flotilla de Aeronaves y la Flotilla de Medidas Contra Minas.

- La Flotilla de Submarinos, con base en el Arsenal de Cartagena, está compuesta por ocho submarinos, cuatro de la clase Delfín y cuatro de la clase Galerna, al mando de un Capitán de Navío. El cometido principal de estas unidades es negar el uso del mar a un potencial enemigo, así como proporcionar los medios necesarios para llevar a cabo misiones de inteligencia con la máxima discreción. Entre sus cometidos secundarios, pero de gran importancia en la actualidad, se encuentran la infiltración de unidades de operaciones especiales y su presencia en aguas potencialmente hostiles en situaciones de crisis, aprovechando su capacidad para situar discretamente toda clase de sensores en las proximidades del foco de la crisis.

Dentro del Arsenal de Cartagena, y en la Base de Submarinos, donde están las instalaciones especificas para el mantenimiento de estos buques, está ubicada también la Escuela de Submarinos, creada para la instrucción y adiestramiento de las dotaciones submarinistas, con unos simuladores en los que se reproducen fielmente los equipos y el comportamiento de las cámaras de mando, y central de máquinas de los submarinos, pudiendo generar en ellos cualquier situación operativa y muy especialmente las emergencias en la mar.

- La Flotilla de Aeronaves, igualmente al mando de un Capitán de Navío, comprende todos los medios aéreos de la Armada. Está compuesta por 57 unidades, que se distribuyen en 6 escuadrillas, cada una de las cuales agrupa aeronaves del mismo tipo:

- La $3^{\mathrm{a}}$ Escuadrilla está compuesta por 10 helicópteros de ataque y transporte de tropas $A B-212$, que se emplean principalmente como helicópteros de asalto en operaciones anfibias.

- La $4^{\text {a }}$ Escuadrilla está constituida por 3 aviones Cessna Citation de media velocidad. Se utilizan en misiones de patrulla marítima y apoyo logístico.

- La $5^{\mathrm{a}}$ Escuadrilla está formada por 11 helicópteros Sikorsky $S H-3 D$ Sea King que, gracias a su sonar calable a profundidad variable, constituyen un elemento importante de la capacidad 
antisubmarina de la Flota. Tres de ellos han sido transformados y equipados con un radar de alerta aérea Search Water que proporcionan al Príncipe de Asturias la posibilidad de detectar aeronaves aproximándose a baja cota. Está previsto que en un futuro próximo las unidades con versión antisubmarina sean transformadas en helicópteros de transporte de tropas de Infantería de Marina para, de esta forma, cubrir una demanda derivada de la situación estratégica actual.

- La $6^{a}$ Escuadrilla está formada por 10 helicópteros ligeros Hughes 500 dedicados a la formación y el adiestramiento de pilotos.

- La $9^{a}$ Escuadrilla comprende 17 aviones $A V-8 B$ y $B+$ Harrier de despegue vertical, que proporcionan el elemento principal de proyección del poder naval, así como un notable incremento de la capacidad de defensa aérea de los buques.

- La 10 Escuadrilla, con 6 helicópteros SH-60 Sea Hawk, concebidos como parte indivisible de las fragatas clase Santa María, es donde descansa una parte esencial de la capacidad antisubmarina y antisuperficie de estos buques.

La Flotilla de Aeronaves se encuentra ubicada en la Base Naval de Rota, donde también dispone de instalaciones para el mantenimiento de las aeronaves, así como de la Escuela de Dotaciones Aeronavales $E D A N$ para la formación e instrucción de su personal de vuelo, con simuladores de los aviones AV-B y helicópteros SH-60 que permiten rentabilizar notablemente el esfuerzo de vuelo real de las aeronaves. También es responsable de la formación de los controladores tácticos de aeronaves que operan desde los buques en la mar.

- La Flotilla de Medidas Contra Minas, incorporada recientemente a la Flota, comprende todos los medios de la Armada de guerra de minas. Está mandada por un Capitán de Navío y tiene su base en Cartagena. Tiene como misión principal mantener una capacidad en guerra de minas para poder actuar en las zonas de interés estratégico español que permita la libertad de navegación del tráfico marítimo, así como apoyar acciones cerca de la costa de un grupo de combate o una fuerza anfibia operativa, asumiendo al mismo tiempo nuestros compromisos con la defensa colectiva en aquellos escenarios de interés común.

La Flotilla de $M C M$ está compuesta por la $1^{\text {a }}$ Escuadrilla, con

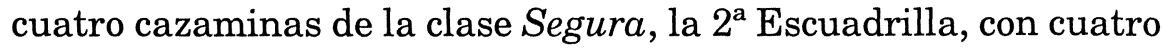


dragaminas de la clase Júcar, el buque de mando y apoyo Diana y la Unidad de Buceadores de Medidas Contraminas UBMCM. Además de estas unidades, la Flotilla de $M C M$ cuenta como elementos de apoyo con el Organismo de Valoración de Adiestramiento de Dragaminas $O V A D$, el centro de datos de guerra de minas, y las correspondientes instalaciones de mantenimiento, todos ellos dentro del Arsenal de Cartagena.

- Las Escuadrillas, también en número de tres, son unidades de composición homogénea, convenientes para facilitar el adiestramiento y, sobre todo, para rentabilizar el mantenimiento y el aprovisionamiento de buques de la misma clase. Están todas ellas mandadas por Capitanes de Navío.

- La $21^{\mathrm{a}}$ Escuadrilla, con base en Cartagena, está formada por cuatro corbetas de la clase Descubierta. Son buques de 1.600 ton. bien armados con torpedos $M k-46$ y cohetes antisubmarinos Bofors, misiles antibuque Harpoon, misiles antiaéreos Aspide, cañones de $76 \mathrm{~mm}$ OTO Melara y de $40 \mathrm{~mm}$ Bofors. A pesar de su pequeño desplazamiento son buques muy valiosos como escoltas.

- La $31^{\mathrm{a}}$ Escuadrilla, basada en el Arsenal de El Ferrol, comprende las 5 fragatas clase Baleares de 4.000 ton. que, aunque se encuentran en su ultimo tercio de vida, mantienen su eficacia gracias a las modernizaciones que se han llevado a cabo. Su capacidad operativa es muy equilibrada, destacando su radar aéreo tridimensional SPS-52, que las capacita para el control e interceptación de aeronaves. Son además, y hasta la entrada en servicio de las F-100, los únicos buques dotados de un cañón de $127 \mathrm{~mm}$, idóneo para el fuego naval de apoyo. Cuentan además con misiles Harpoon y Standard contra blancos de superficie y aeronaves.

- La $41^{a}$ Escuadrilla, basada en Rota, está formada por seis modernas fragatas de la clase Santa María de 4.000 ton. Tienen una gran capacidad antisubmarina, que le proporciona el sistema LAMPS con el helicóptero Sea Hawk y el sonar pasivo remolcado TACTAS, sin olvidar su capacidad antisuperficie y antiaérea, gracias a sus misiles Harpoon y Standard.

\section{Unidades independientes}

Son aquellas unidades singulares que tienen una dependencia directa del ALFLOT: los Buques Logísticos. La Armada cuenta con el buque de aprovisionamiento de combate Patiño, diseñado en conjunción 
con la marina holandesa, que proporciona apoyo logístico en forma de combustible, agua, municiones, repuestos, sanidad y víveres, y el petrolero de flota Marques de la Ensenada, que asegura el suministro de combustible.

\section{La Brigada de Infantería de Marina, BRIMAR}

Las fuerzas del «Tercio de Armada» de Infantería de Marina se integran en la Flota con una dependencia especial. Por un lado dependen orgánicamente del Comandante General de la Infantería de Marina; y por otro, sus fuerzas están asignadas en permanencia al ALFLOT para la preparación y el adiestramiento de la Fuerza de Desembarco. Además, el ALFLOT ejerce la potestad disciplinaria y gestiona parte de sus recursos económicos y administrativos.

Se encuentran en San Fernando, Cádiz, al mando de un General de Brigada de Infantería de Marina. Su entidad es la de una Brigada de Infantería de Marina BRIMAR, con dos batallones de Infantería de Marina, un batallón mecanizado, un grupo de artillería, una Unidad de Operaciones Especiales $U O E$ y un grupo de apoyo de servicios de combate.

La BRIMAR, con unos efectivos de 4.000 personas, es una unidad de alta disponibilidad y absolutamente autónoma. Tiene unas características que la hacen diferente del resto de las brigadas de las FAS; y es que aparte de su capacidad de asalto anfibio, posee una gran potencia de fuego y una específica movilidad, la que le proporcionan todos sus vehículos, especialmente preparados para el vadeo.

La $B R I M A R$, gracias a estas capacidades y al alto espíritu y preparación de sus miembros, hace honor al lema de la Infantería de Marina española Valientes por tierra y por mar.

\section{El apoyo a la Fuerza}

Está constituido por el conjunto de órganos que auxilian al ALFLOT en el adiestramiento, preparación y evaluación de las unidades de la Flota. Dichos órganos son:

- El CEVACO, o Centro de Evaluación para el Combate, cuyo cometido es el de evaluar el rendimiento y la eficacia de los buques, colaborando en la obtención de la apropiada Calificación Operativa para el Combate o CALOPCO. Además cuenta con el Centro de Seguridad Operativa de la Armada, donde se investigan y dan normas a todos los buques, unidades e insta- 
laciones navales, para evitar toda clase de accidentes relacionados con el servicio

El ALFLOT asigna cuatro niveles diferentes de calificación operativa, de acuerdo con las recomendaciones del $C E V A C O$ y las notas obtenidas en las CALOPCO:

$\mathrm{N}$ - Negativa. El buque no está listo para navegar debido a estar sometido a obras prolongadas o en situación de reserva. A- Paz-Adiestramiento/Comisiones. El buque no está listo para combatir porque necesita acciones de mantenimiento importantes, completar su personal o mejorar su adiestramiento.

O- Paz-Operaciones, cuando existe alguna limitación concreta que impide al buque emplear plenamente su capacidad de combate. C- Combate, establece que el buque está listo para combatir.

- Los Organismos de Valoración y Adiestramiento de Submarinos OVAS y de Dragaminas OVAD ya citados, cuyo cometido es valorar el adiestramiento de este tipo de unidades, de características y misiones muy específicas, y que mantienen una dependencia funcional del CEVACO.

- El Centro de Programas Tácticos y Centro de Instrucción y Adiestramiento o CPT-CIA, es a la vez un centro de instrucción y adiestramiento en el uso de los sistemas de combate de los buques de superficie y un centro técnico encargado del mantenimiento del software de los sistemas de combate de dichos buques. En sus simuladores se puede representar cualquier escenario táctico común, y gracias a un enlace por el circuito Link 11 se puede hacer partícipe del ejercicio a los buques presentes en la bahía y a los simuladores de la Flotilla de Aeronaves.

- El Centro Integrado de Gestión Administrativa, CIGAFLOT, integra los Centros de Comunicaciones, el Centro de Apoyo Informático y los Sistemas de Mando y Control. Su misión principal es la gestión y administración de los sistemas de tecnologías de la información.

\section{Capacidades}

El conjunto de todas las unidades mencionadas representa las posibilidades reales de la Flota, basadas en cuatro capacidades básicas:

- La capacidad de proyección, que se sustenta en el portaaviones Príncipe de Asturias con la Unidad Aérea Embarcada UNAEMB (aviones y helicópteros), los buques anfibios y la Fuerza de Desembarco. Incluye así las capacidades aeronaval y anfibia, que 
permiten proyectar el poder naval mediante la ejecución de cualquiera de las modalidades de una operación anfibia clásica, y cualquier tipo de operaciones de paz, evacuación de no combatientes $N E O$, ayuda humanitaria, etc.

- La capacidad de protección a fuerzas navales y tropas operando en tierra -basada en los escoltas-, así como a las líneas de comunicación del tráfico marítimo, propio y aliado $\mathrm{y}$, cuando sea necesario, colaborar con otras autoridades nacionales en la protección de nuestros intereses nacionales en el ámbito marítimo (pesqueros, explotaciones off-shore etc.)

- La capacidad de apoyo logístico operativo, basada en los buques logísticos.

- La capacidad para garantizar la libertad de acción de nuestras fuerzas navales, especialmente en los escenarios litorales alejados, basada en los submarinos y los cazaminas.

\section{Estructura operativa}

Para llevar a cabo los diferentes tipos de operaciones se constituyen agrupaciones operativas, bajo las órdenes del mando que en cada caso se designe. Estas agrupaciones tienen carácter temporal y se establecen mediante directivas u órdenes, en las cuales se fija la misión, se designa al mando y su estructura, y se asignan los medios para cumplirla.

En el seno de la Flota dos son los mandos operativos alrededor de los cuales normalmente se crean estas organizaciones operativas, el COMARFOR y el COMGRUFLOT.

- El COMARFOR, Comandante del Cuartel General Marítimo Español de Alta Disponibilidad, que es -llegado el caso- el mando operativo natural de una fuerza naval española de una cierta entidad, para operaciones reales o ejercicios tanto nacionales como internacionales.

- El COMGRUFLOT, Comandante del Grupo de Unidades de Proyección, que es el mando operativo natural de una agrupación naval española, de un nivel inferior al que mande el COMARFOR, y que normalmente tiene como objetivo la proyección del poder naval sobre tierra.

\section{Preparación}

La preparación de la Fuerza, es el conjunto de actividades dirigidas a adiestrar, evaluar y alistar a las unidades que la componen para 
que puedan cumplir eficazmente sus cometidos operativos. Estas actividades se llevan a cabo en la estructura orgánica y en éllas se tendrán en cuenta los cometidos exigibles a nuestras fuerzas navales tanto en el plano nacional como en el internacional,

Las actividades se programan de forma secuencial, en un proceso que va de menos a más denominado Ciclo de Actividad Progresiva, que normalmente se inicia después de un período de inmovilización por obras de mantenimiento y que se puede sintetizar en las siguientes fases:

- Alistamiento individual

En él la unidad debe comprobar el correcto funcionamiento de todos los sistemas del buque y elevar el nivel de instrucción y adiestramiento de la dotación, haciendo el mayor uso posible de los centros de adiestramiento. Está dirigido por el comandante del buque, pero las propuestas de programación, la determinación de necesidades y el control, es responsabilidad de los comandantes de las agrupaciones orgánicas.

- Calificación operativa

Orientada a progresar en la preparación conseguida en el alistamiento individual, apoyar el adiestramiento y evaluar el grado de preparación de una unidad para el combate.

- Adiestramiento colectivo básico

Se desarrolla normalmente en el seno de las agrupaciones orgánicas y está orientado a la aplicación de la táctica naval correspondiente a cada tipo y clase de buques, una vez que finaliza un período de mantenimiento. Es dirigido y controlado por los comandantes de las agrupaciones orgánicas.

- Adiestramiento colectivo avanzado

Se desarrolla en el seno de agrupaciones operativas temporales creadas para la participación en ejercicios nacionales o internacionales de alto nivel. Normalmente se realiza en cualquier zona dentro del área de interés nacional próximo a nuestras costas, mediante diversos ejercicios.

\section{Participación en ejercicios}

Centrándonos en la última fase del Ciclo de Actividad Progresiva, el adiestramiento avanzado se lleva a cabo mediante la participación en ejercicios nacionales e internacionales. 


\section{Ejercicios nacionales}

El adiestramiento se realiza en cualquier zona dentro del área de interés nacional próxima a nuestras costas, mediante diversos ejercicios, entre los que cabe destacar:

- Los que tienen un carácter aeronaval

- Los específicos de la fuerza anfibia

- Los propios de la Infantería de Marina.

- El Neotapón, que es un ejercicio nacional de carácter anual, en el que se invita a las marinas aliadas, y se desarrolla en la zona del Estrecho de Gibraltar, el mar de Alborán y el Océano Atlántico, incluyendo en ocasiones también a las Islas Canarias. El principal objetivo del ejercicio es practicar el control del Estrecho, sus accesos y el espacio aéreo comprendido. Tiene el carácter de ejercicio marítimo, aunque con una significativa participación de aviones del Ejército del Aire y diferentes unidades del Ejército de Tierra, especialmente de la artillería de costa del Estrecho.

Además de estos ejercicios de carácter predominantemente naval, también dentro del ámbito nacional se realizan otros de carácter conjunto.

La necesidad de impulsar la acción conjunta en la actualidad no sólo es debida a la obligación de lograr una mayor economía de medios sino también, y en la misma medida, para conseguir una mayor eficacia al actuar con otros ejércitos. La capacidad actual de armas y sensores con influencia en campos de batalla donde operan otros ejércitos, obliga a operar conjuntamente para obtener mejores resultados.

- Ejército de Tierra

Con el Ejército de Tierra la participación es la siguiente:

- Unidades de Infantería de Marina en ejercicios de carácter general para poner en práctica procedimientos comunes

- Transportes de tropas y material

- Colaboración con las Fuerzas Aeromóviles FAMET, para el adiestramiento de las Unidades de Operaciones Especiales

- Buceadores de combate

- En el campo de las operaciones especiales colaboran submarinos y buques.

- En ejercicios de defensa aérea de la Base Naval de Rota.

- Ejercicios con el Mando de Artillería de Costa, para el control del Estrecho de Gibraltar 
- Colaboración con las Fuerzas Aeromóviles FAMET, para el adiestramiento de sus dotaciones.

- Ejército del Aire

En este caso la colaboración y participación se dirige sobre todo a ejercicios de defensa aérea en los que intervienen aviones y buques de superficie. Se prevé la participación futura en estos ejercicios de las fragatas $F-100$, como una extensión de la capacidad de detección aérea en profundidad y defensa aérea del territorio.

Igualmente la participación de los aviones de patrulla marítima $P-3 B$ Orion, bajo el control operativo del ALFLOT, es vital para el control de extensas áreas marítimas.

\section{Ejercicios internacionales}

La participación de las unidades de la Flota en ejercicios con otras naciones, puede ser dentro del contexto de la OTAN o bien en el ámbito bilateral, soportados estos últimos por los correspondientes acuerdos técnicos.

Dentro del primer grupo podemos destacar:

- En el Atlántico, el STRONG RESOLVE de SACLANT, NORTHERN LIGHT de CINCEASTLANT y LINKED SEAS de CINCSOUTHLANT.

- En el Mediterráneo, el DYNAMIC MIX de CINCSOUTH, DESTINED GLORY de COMSTRIKFORSOUTH y DOG FISH de COMSUBSOUTH.

En el ámbito bilateral son reseñables: el ejercicio EOLO, con Francia, Italia y Portugal: el GENERAL SHARK, PHIBLEX y LISA AZUL con la marina de los EEUU; MECO-RECO y FANFLOT con Francia; MAES y MEDATEX con Marruecos, COMTEX y SWORDFISH con Portugal: JMC y ASWX con el Reino Unido; NIRIIS con Grecia; DOGU AKDENIZ con Turquía; y FALCON NUT con Holanda.

Por último cabe destacar el ejercicio UNITAS, que se desarrolla en aguas sudamericanas y en el que participan las marinas de Argentina, Brasil, EEUU y Uruguay.

\section{Ámbitos de actuación}

La decisión de compartir la seguridad con nuestros aliados y la movilidad, rapidez de despliegue y autosuficiencia logística, características fundamentales e intrínsecas de la Fuerza Naval, aconsejan 
que el cometido primordial de la Flota -la preparación de sus unidadesresponda a los escenarios más probables de acción, traducidos en los siguientes despliegues básicos:

- Actuación como componente naval de una operación conjunta en el ámbito nacional, o combinada en el seno de la OTAN o de la Unión Europea.

- Integración permanente o periódica en las fuerzas marítimas de alta disponibilidad de la OTAN, en el Mediterráneo, Atlántico y de MCM.

- Participación en fuerzas similares, que puedan crearse, en el ámbito exclusivamente europeo.

- Presencia naval española en el continente americano mediante despliegues esporádicos.

- Colaboración con otros organismos del Estado, con las Fuerzas y Cuerpos de Seguridad, y en el apoyo a las autoridades civiles nacionales o extranjeras.

\section{Contribución a la Alianza y a Fuerzas Multinacionales}

Como ya se ha mencionado anteriormente, la decisión de compartir la seguridad con nuestros aliados nos obliga a preparar a nuestras unidades para que sean compatibles e interoperables con los procedimientos de trabajo y equipos de las marinas amigas, y poder llevar a cabo un despliegue, ya sea con carácter permanente o temporal. Esto se traduce para nuestras unidades en las siguientes operaciones posibles:

- Actuación como componente naval en una operación conjunta y combinada en el seno de la Alianza Atlántica o de la Unión Europea. Se contempla como posibilidad alternativa la de actuar como fuerza exclusivamente marítima en los mismos ámbitos.

- Participación permanente o periódica en las Fuerzas Marítimas de Reacción Inmediata de la OTAN en el Atlántico STANAVFORLANT, en el Mediterráneo STANAVFORMED, de medidas contra minas del sur de Europa MCM FORSOUTH, y previsión de participación en fuerzas similares en el ámbito exclusivamente europeo EUROMARFOR y SIAF.

\section{Participación en operaciones reales}

Las operaciones reales en las que ha participado la Flota en los últimos años, además de las de vigilancia y obtención de inteligencia, 
se han desarrollado en escenarios alejados de España como son el Golfo Pérsico, ex-Yugoslavia, Kosovo, Albania y Centroamérica.

- Guerra del Golfo

La Flota participó durante todo el conflicto, con dos corbetas asignadas a las operaciones de embargo en el Mar Rojo y con una fragata de la $41^{\mathrm{a}}$ Escuadrilla en el Golfo Pérsico.

- Ex-Yugoslavia

Durante las operaciones en el Adriático, la Flota colaboró con dos fragatas de manera permanente, una de ellas integrada en la agrupación de la OTAN y la otra en el Grupo de la Unión Europea Occidental, y con un submarino y un buque logístico de forma periódica. Al mismo tiempo los buques anfibios fueron empleados en el transporte de tropas y material de Infantería de Marina y del Ejército de Tierra.

La Brigada de Infantería de Marina ha participado, y sigue haciéndolo, mediante un subgrupo táctico integrado en la brigada española desplegada en Bosnia, dentro de las Fuerzas de Estabilización SFOR. Este subgrupo es relevado cada seis meses.

- Albania

En este caso los buques anfibios contribuyeron al despliegue y posterior repliegue del Grupo Táctico de La Legión Serranía de Ronda, como fuerza de la. ONU participante en la operación Alba.

- Operación de ayuda humanitaria a Centroamérica

Consistió en proporcionar ayuda para la reconstrucción en Guatemala, Honduras, Nicaragua y El Salvador, después del devastador paso del huracán Mitch por Centroamérica. Esta operación fue realizada desde el 22 de noviembre del año 1998 hasta el mes de abril de 1999, y participaron tres buques anfibios.

En esta operación hay que destacar la actuación de los servicios médicos de los buques, que asistieron a más de 5.000 personas, de ellas 3.500 niños. También se transportaron más de 2.000 toneladas de material de ayuda humanitaria, y un puente tipo Bayle del Ejército de Tierra para restablecer el paso del río Guasaule entre Honduras y Nicaragua.

- Kosovo

Los buques anfibios participaron en la operación para proporcionar ayuda humanitaria a los refugiados kosovares. Durante la operación Alfa Kilo estuvieron operando en el Adriático las agrupaciones de la OTAN, en cada una de las cuales estaba integrada una fragata española. 
Además, los dos buques logísticos estuvieron temporalmente integrados en estas agrupaciones, mientras que los buques anfibios estuvieron involucrados en el transporte de tropas y material con destino a las fuerzas de la OTAN que operaban en Kosovo.

- Guerra contra el terrorismo internacional

Desde el pasado 9 de octubre del 2001, en respuesta a la invocación del artículo $\mathrm{V}$ del Tratado de Washington, y en apoyo a la operación Libertad Duradera, dos fragatas españolas de forma permanente, y un buque logístico periódicamente, participan en operaciones de vigilancia y presencia naval en el Mediterráneo Oriental, integradas en las agrupaciones permanentes de la OTAN.

Por otra parte, además de las unidades anteriores, y desde el mes de marzo del presente año 2002, otras dos fragatas y un buque de apoyo logístico se encuentran en el Océano Indico participando en las operaciones contra el terrorismo internacional que, lideradas por los EEUU, se están llevando a cabo en esa zona de conflicto.

\section{Conclusiones}

A lo largo de estas líneas se ha descrito la organización, composición y cometidos de la Flota. No olvidemos que sus efectivos, 12.400 personas, suponen la mitad de la Armada Española, que sus 57 aeronaves son todas las que vuelan con la corona y el ancla y que sus 39 buques prácticamente representan el 90\% del tonelaje total de los buques de la Armada.

El resultado de todos estos medios supone un conjunto equilibrado, con buques de superficie, submarinos y aeronaves. La Flota española pertenece a un reducido club de marinas oceánicas que, como la estadounidense, británica, francesa, italiana, rusa e india poseen portaaviones.

Igualmente podemos estar orgullosos de poseer la Infantería de Marina más antigua del mundo, y la más potente de Europa, con unos buques anfibios, Galicia y Castilla, que son envidiados en nuestro entorno.

El futuro de esta Flota está marcado por las nuevas construcciones y adquisiciones, pues no hay que olvidar que hay una serie de unidades que se encuentran en su último tercio de vida.

Actualmente se está desarrollando el programa de construcción de cuatro fragatas clase Alvaro de Bazán (F-100), posiblemente las mejores en su categoría. Igualmente se está llevando a cabo la consecución de la segunda serie de cazaminas clase Segura, con dos unidades que relevarán a los veteranos dragaminas costeros con más de 40 años 
en sus cuadernas, pero todavía operativos y animosos. Y la adquisición de vehículos acorazados Piraña proporcionarán una nueva movilidad en tierra a la Infantería de Marina.

En el aire queda la construcción de los submarinos Serie 80, que serán el relevo de los veteranos Delfín, a punto de doblar el tercer decenio, y la construcción de un tercer buque anfibio, posiblemente un $L P H$ con cubierta corrida para operaciones con aviones de despegue vertical. El reemplazo de los helicópteros Sea King, actualmente en vías de remodelación hacia cometidos de transporte de combate, es otra signatura pendiente. $\mathrm{Y}$ así podríamos seguir relatando todo un programa naval, condicionado siempre a las disponibilidades presupuestarias.

Pero no todo son equipos y buques, que de nada valdrían sin unas dotaciones bien adiestradas y motivadas. La profesionalización, realizada en tan corto período de tiempo, ha supuesto todo un reto, así como el tener que realizar el mismo trabajo en las unidades con menos personal, hasta que se estabilice el ingreso de los nuevos marineros y soldados profesionales. Para ello ha sido necesario adoptar un nuevo estilo de mando, más acorde con el sentir de la juventud actual, y practicar a todos los niveles una nueva asignatura, la «motivación», sin la cual sería difícil conseguir una máquina perfectamente engrasada, para evitar el óxido del desánimo y el abandono.

En esencia, España puede enorgullecerse de poseer una Flota moderna, de un tamaño adecuado a nuestro entorno y a los riesgos de la situación mundial, pero no hay que olvidar que lo que hoy poseemos ha sido el trabajo de las generaciones que nos precedieron, y que es nuestra obligación que esa institución secular que es la Armada perdure en el grado de eficacia que nos exige España y su Defensa. 